\title{
Haemodynamic response to myocardial ischaemia during unrestricted activity, exercise testing, and atrial pacing assessed by ambulatory pulmonary artery pressure monitoring
}

\author{
RICHARD D LEVY, LEONARD M SHAPIRO, CHRISTINE WRIGHT, \\ LORNA MOCKUS, KIM M FOX \\ From the National Heart Hospital, London
}

SUMMARY Ambulatory pulmonary artery pressure monitoring by means of a transducer tipped catheter with a simultaneous frequency modulated electrocardiogram and a miniaturised tape recorder was used to study the haemodynamic implications of ST segment depression in patients with coronary artery disease. Nineteen male patients (mean (SD) age 58 (11) years) with clinical and angiographic evidence of coronary artery disease were studied together with six controls. Changes in the ST segment and pulmonary artery diastolic pressure during treadmill exercise, atrial pacing, and unrestricted ambulant activity were analysed. During exercise, pulmonary artery diastolic pressure rose significantly in patients with coronary artery disease but not in the controls. One patient with ST depression $>1 \mathrm{~mm}$ did not have a rise in pulmonary artery diastolic pressure on exercise; two had a rise in pulmonary artery diastolic pressure with no ST segment change despite severe angina. The pulmonary artery diastolic pressure tended to rise before or simultaneously with the onset of ST segment depression. The haemodynamic response to atrial pacing was similar in normal controls and patients with coronary artery disease. During ambulatory monitoring there were 29 episodes of ST segment depression all of which were associated with a rise in pulmonary artery diastolic pressure and chest pain. The onset of ST segment depression occurred before a rise in pulmonary artery diastolic pressure in 11 episodes, was simultaneous with it in 11, and followed it in seven episodes. During exercise and ambulatory monitoring there was a correlation between the magnitude of ST segment depression and the rise in pulmonary artery diastolic pressure. Pain was a late feature during exercise, atrial pacing, and anginal episodes.

This technique for the first time allows the relation between ST segment changes and haemodynamic alterations in left ventricular function to be assessed in ambulant patients with coronary artery disease.

During angina pectoris there is a rise in left ventricular filling pressure that causes an increase in pulmonary artery wedge pressure and pulmonary artery diastolic pressure. ${ }^{1}$ Previous studies of the haemodynamic changes in patients with angina have

Requests for reprints to Dr Richard D Levy, National Heart Hospital, Westmoreland Street, London W1M 8BA.

Accepted for publication 18 March 1986 used fluid filled catheters and external transducers and have all been based either in the catheter laboratory or in the coronary care unit. ${ }^{23}$ In these studies the changes in left ventricular end diastolic pressure have been documented in exertional angina ${ }^{4}$ and during atrial pacing ${ }^{256}$ but in none of these studies has ambulatory recording been performed and the exact relation of changes in the ST segment to the onset of haemodynamic alterations has not been established. 
We have applied the technique of ambulatory pulmonary artery pressure monitoring by a transducer tipped catheter and a simple recording system to study the haemodynamic changes during treadmill exercise, atrial pacing, and unrestricted daily activity in patients with coronary artery disease and in normal controls.

\section{Patients and methods}

Nineteen male patients (mean (SD) age 58 (11) years) with clinical and angiographic evidence of coronary artery disease were studied. All had a positive exercise test and nine had had a previous myocardial infarction (eight inferior, one anterior). Fifteen had three vessel coronary disease, five with a left main stem stenosis; three had two vessel disease; and one had single vessel disease. All patients were shown to have at least one episode of ST segment depression on ambulatory monitoring over a 24 hour period. In addition, a further six patients with chest pain, normal coronary arteries, and negative exercise tests were studied and acted as controls.

Patients with evidence of heart failure and other forms of heart disease including pulmonary vascular disease were excluded. All antianginal medication other than glyceryl trinitrate was discontinued at least 48 hours before the study and pulmonary artery pressure monitoring was performed for at least 24 hours and where possible for up to 48 hours. Frequency modulated ST segment monitoring was performed throughout this period.

The study was approved by the ethics committee and patients gave their informed and written consent.

The transducer tipped pulmonary artery catheter was introduced percutaneously via a subclavian vein to a proximal pulmonary artery under fluoroscopy in the cardiac catheter laboratory at the time the patient had cardiac catheterisation. ${ }^{7}$ In 17 patients atrial pacing was performed at this time. Patients were then encouraged to be as active as possible within the confines of the hospital. In 15 patients an exercise test was performed two hours before withdrawal of the pulmonary artery catheter.

Pulmonary artery pressure monitoring-A polyurethane six French NIH type catheter with a miniature strain gauge transducer mounted on the tip was used. This was immersed in saline for one hour before use and was calibrated. The transducer was driven and demodulated by an electrically isolated Gaeltec pre-amplifier. The pulmonary artery pressure was recorded on an Oxford Medilog I miniature tape recorder which had been modified by the insertion of an AM4 pressure module.

At the end of 24-48 hours recording the pul- monary artery catheter was removed and the pulmonary artery pressure trace was replayed via a PB2 unit and a PM3 amplifier. ${ }^{7}$ This was displayed on an SE laboratories 6008 oscillograph. The entire period of recording of the pulmonary artery diastolic pressure was displayed on ultraviolet paper and measured by means of a calibrated scale from a zero reference point. The pulmonary artery diastolic pressure was measured during both painful and painless episodes of ST segment depression on a beat to beat basis five minutes before the onset of the earliest change in pulmonary artery pressure and for five minutes after the pulmonary artery pressure had returned to baseline. The pulmonary artery diastolic pressure was measured at the same point in each respiratory cycle.

$S T$ segment monitoring was performed with a frequency modulated recorder and leads CM2 and CM5 were recorded. The electrocardiogram was replayed on an Oxford MA20 scanner. Changes in the ST segment were measured $80 \mathrm{~ms}$ after the J point to an accuracy of $0.1 \mathrm{~mm}$ by means of a magnifying lens with graticule. Planar or downsloping ST segment depression of $>1 \mathrm{~mm}$ that lasted at least $30 \mathrm{~s}$ was considered to be significant. The heart rate and ST segment changes were analysed on a beat to beat basis for five minutes before each episode and again up to five minutes after the ST segment had returned to basal levels. The pulmonary artery recorder was linked to the frequency modulated electrocardiogram by a patient event button.

Exercise test-Patients performed an exercise test according to a modified Bruce protocol. ${ }^{8}$ Transducer tipped pulmonary artery pressure and the frequency modulated electrocardiogram were recorded continuously. A 12 lead electrocardiogram was recorded on paper at one minute intervals. The exercise test was terminated by anginal pain, dyspnoea, multiple ventricular extrasystoles, hypotension, ST segment depression $\geqslant 0.3 \mathrm{mV}$, or exhaustion.

Atrial pacing-A bipolar electrode was introduced via the right femoral vein to the right atrium. Control measurements of the resting 12 lead electrocardiogram were obtained and the heart rate was increased by 10 beats a minute, with each rate maintained for 60 seconds. Atrial pacing was discontinued if the patient complained of angina, if ST segment depression was $>1 \mathrm{~mm}$, or a rate of 160 beats per minute was reached. ST segment depression was measured at $80 \mathrm{~ms}$ after the J point. The 12 lead electrocardiogram together with the pulmonary artery changes and ST segment changes on Holter monitoring were measured until the heart rate returned to the baseline levels.

Angina diary-Patients kept diaries during the 


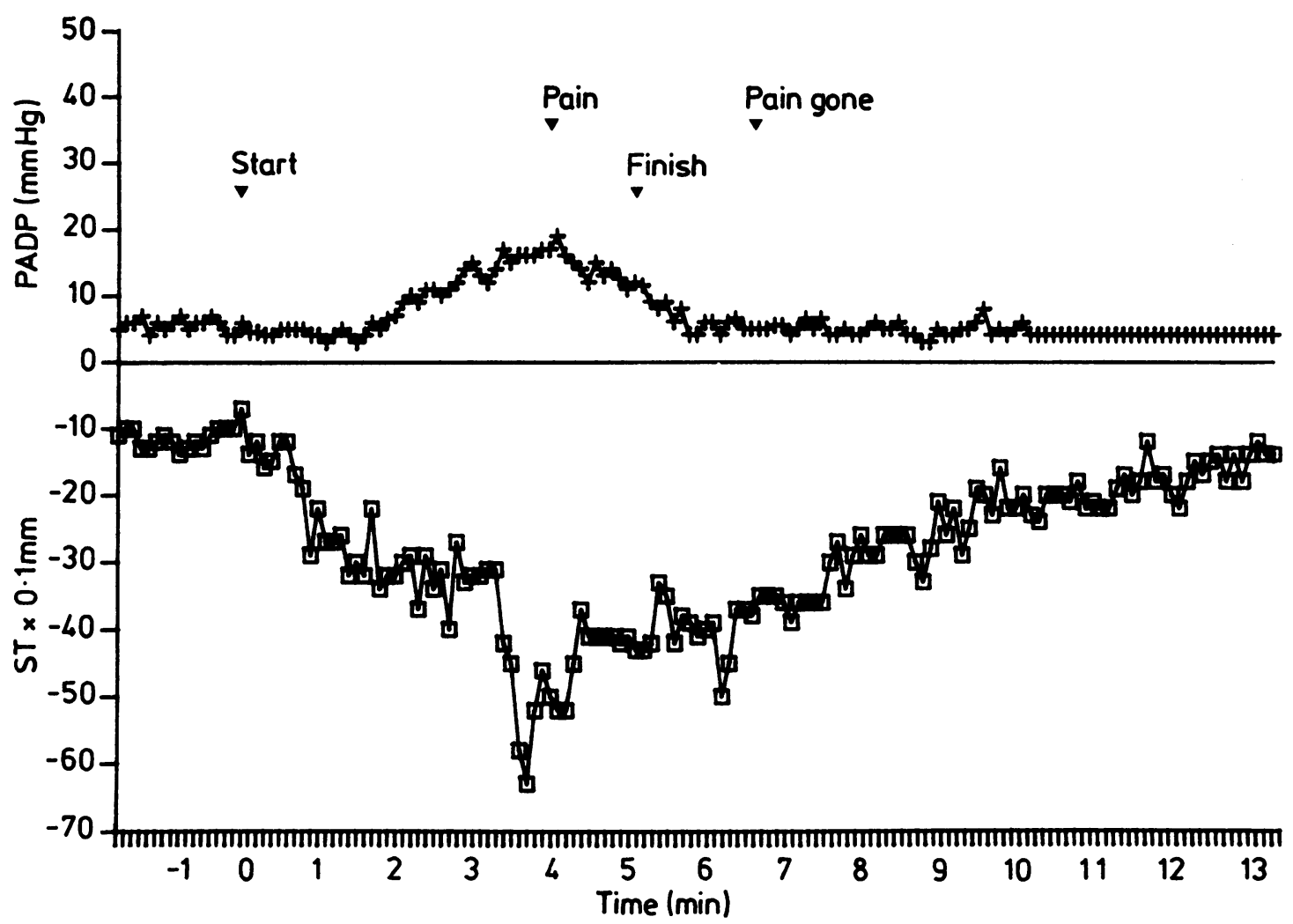

Fig. 1 Changes in pulmonary artery diastolic pressure (PADP) and the $S T$ segment during a treadmill exercise test in a patient with coronary artery disease. Heart rate increased from 90 to 120 beats/min and the patient performed 5 minutes of exercise on a modified Bruce protocol.

period that ambulatory pulmonary artery pressure and the ST segment were monitored; they noted the time of onset and severity of chest discomfort and activity at the time of the pain. Patients were asked to press the event marker during chest pain.

STATISTICAL ANALYSIS

A change in the basal ST segment, heart rate, or pulmonary artery diastolic pressure of at least three standard deviations from the mean was regarded as significant. Wilcoxon rank sum testing was used for analysis. Pearson correlation coefficient was calculated where necessary.

\section{Results}

\section{EXERCISE RESPONSE}

Controls

Five out of the six patients achieved a heart rate of $>150$ beats per minute and at least stage 4 of the modified Bruce protocol (median 13.2 minutes, range 12-16); the remaining female patient reached stage 3 with a heart rate of 132 beats per minute. There was no increase in pulmonary artery diastolic pressure with exercise (median $=0 \mathrm{~mm} \mathrm{Hg}$, range $=0-3)$. There was, however, a rise in systolic pressure (median $15.5 \mathrm{~mm} \mathrm{Hg}$, range 12-34).

\section{Coronary artery disease}

Fifteen of the nineteen patients performed treadmill exercise (Fig. 1). The maximum heart rate achieved was a median 115 beats per minute (range 89-168) and an exercise duration of a median 7.5 minutes (range 3.6-13.8). The pulmonary artery diastolic pressure increased by a median $5.2 \mathrm{~mm} \mathrm{Hg}$ (range $0-13.8)$ on exercise; this was a significant rise compared with baseline and the normal response $(p<0.01)$. In nine patients ST segment depression of $>1 \mathrm{~mm}$ occurred during exercise; eight of these patients had a significant rise in pulmonary artery 


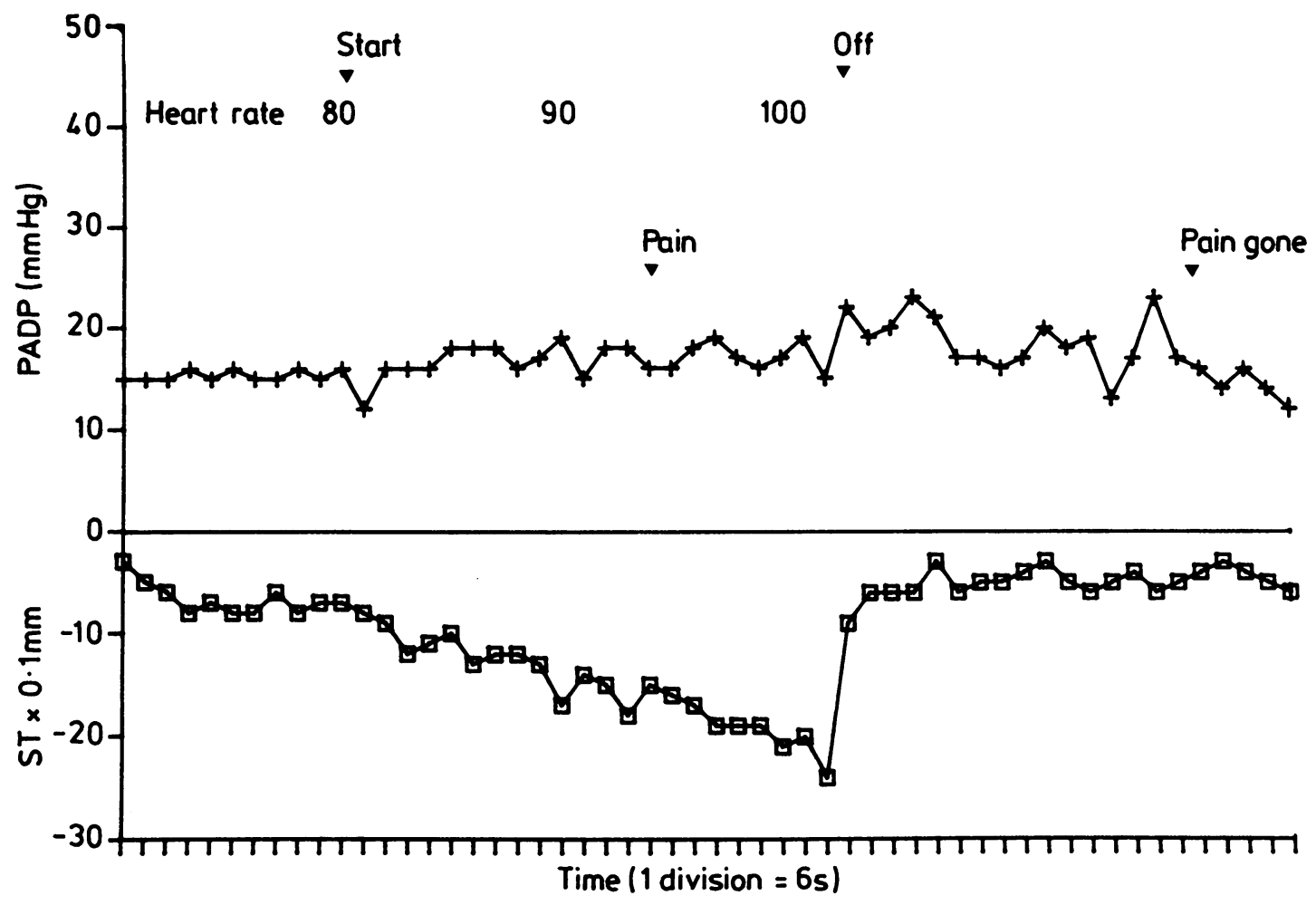

Fig. 2 Changes in pulmonary artery diastolic pressure (PADP) and the ST segment during atrial pacing in a patient with coronary artery disease. The small rise in pulmonary artery diastolic pressure was not significantly different from the slight increase in the control group.

diastolic pressure. The onset of a significant rise in pulmonary artery diastolic pressure preceded ST segment depression in six patients, was simultaneous with it in two, and in one patient it followed the earliest change in the ST segment.

Six patients had no significant ST segment change; five complained of angina pectoris, and four had a significant rise in pulmonary artery diastolic pressure. The remaining patient showed no alteration in the ST segment or significant rise in pulmonary artery diastolic pressure but did complain of chest pain. One patient had no ST segment changes on exercise, did not complain of chest pain, and had no increase in pulmonary artery diastolic pressure despite a history of angina and the presence of important three vessel coronary artery disease.

There was a good correlation $(r=0.8, p<0.001)$ between the magnitude of ST segment depression and the magnitude of increase in pulmonary artery diastolic pressure. Similarly the duration of increase in pulmonary artery diastolic pressure (median 6.3 minutes, range 3.2-8) resembled the duration of ST segment depression (median 6.3 minutes, range 0-36.5).

The changes in the ST segment and pulmonary artery diastolic pressure always preceded the onset of angina which occurred at about the same time (median 4.7 minutes, range 2-13.6) as the maximum increase in pulmonary artery diastolic pressure $(5.4$ minutes, range 2.5-30.5) and maximum ST segment depression (median $7 \cdot 2$ minutes, range 2.2-17.9). The pulmonary artery diastolic pressure returned to basal level more rapidly (36 seconds, range $0 \cdot 2-2 \cdot 5$ minutes) than the ST segment (median 4.5 minutes, range $0-8.2)$ after the end of exercise $(p<0.01)$.

\section{ATRIAL PACING}

Controls

Three of the six controls were paced to 160 beats/min. Of the remaining three patients, two reached 150 beats/min and one 130 beats/min before the development of atrioventricular block. The 


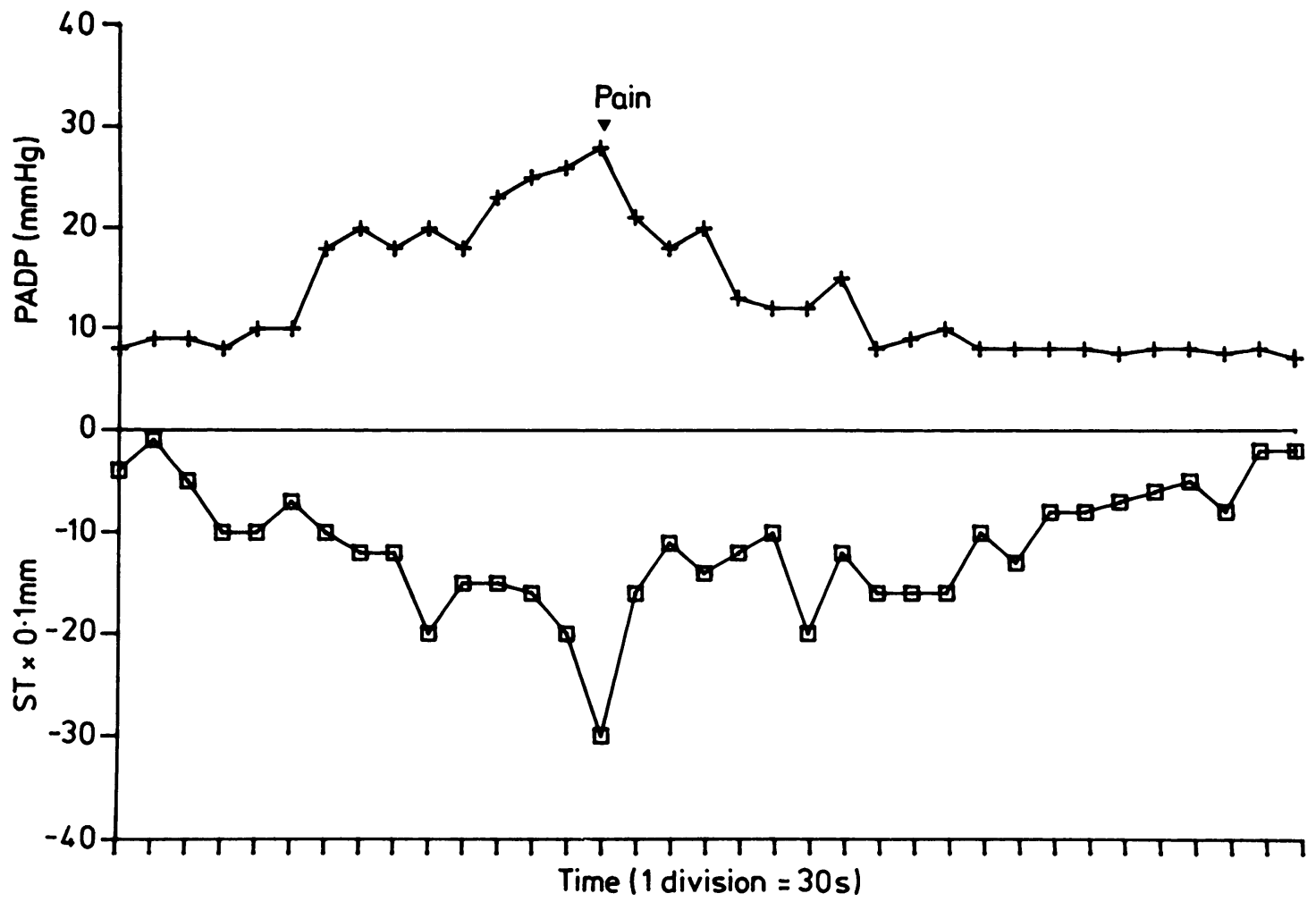

Fig. 3 Changes in pulmonary artery diastolic pressure (PADP) and the ST segment during an episode of angina recorded during ambulatory monitoring.

median increase in pulmonary artery diastolic pressure was $1 \mathrm{~mm} \mathrm{Hg}$ (range 0-5).

Coronary artery disease

Seventeen of the nineteen patients with coronary artery disease were atrially paced; eight patients developed ST segment depression $>1 \mathrm{~mm}$ (Fig.2). Pulmonary artery diastolic pressure rise was not significantly different from the controls (median 4.7 $\mathrm{mm} \mathrm{Hg}$, range 0-9.4).

\section{AMBULATORY MONITORING}

During the period of monitoring the nineteen patients recorded 29 episodes of angina pectoris; all were accompanied by ST segment depression. In all of these episodes there was a significant rise in pulmonary artery diastolic pressure (median $7 \cdot 5$, range 1.8-19.7). The onset of ST segment depression occurred before the rise in pulmonary artery diastolic pressure in 11 episodes, was simultaneous with it in 11, and followed it in the remaining seven episodes. In 28 of the 29 episodes the initial changes in the ST segment and pulmonary artery diastolic pressure preceded the appreciation of chest pain. In 24 of the 29 episodes the maximum ST segment depression and maximum pulmonary artery diastolic pressure rise followed or was simultaneous with the complaint of chest pain. There was a weak correlation between the magnitude of ST segment depression and the magnitude of increase of pulmonary artery diastolic pressure $(r=0.4, p<0.05)$.

\section{Discussion}

During myocardial ischaemia alterations in left ventricular function lead to an increase in end diastolic pressure.$^{4910}$ In the absence of obstructive lesions of the mitral valve and lung disease, these alterations cause a similar rise in pulmonary artery diastolic pressure. $^{71-13}$ Thus ambulatory recording of the pulmonary artery diastolic pressure provides a means of observing changes in left ventricular function while the patient is undergoing his daily activities. In control patients, we found that during 
unrestricted activity and during a formal exercise test there was no acute increase in pulmonary artery diastolic pressure. In contrast, in patients with coronary artery disease there was a significant rise in pulmonary artery diastolic pressure associated with angina during the formal exercise test in 13 of 15 subjects. During unrestricted activity ST segment depression was accompanied by an increase in pulmonary artery diastolic pressure in those with angina. Although exercise testing and unrestricted activity caused an increase in pulmonary artery diastolic pressure their effects differed in other respects. The greatest increases in pulmonary artery diastolic pressure were seen during unrestricted activity. A small increase in pulmonary artery diastolic pressure occurred with atrial pacing in controls and in those with coronary artery disease. Our findings differ from those of a previous study in which there were similar rises in pulmonary artery diastolic pressure in pacing induced and spontaneous angina. ${ }^{14}$ There was no fixed relation between the onset of ST segment changes and the earliest change in pulmonary artery diastolic pressure. During the exercise test, pulmonary artery diastolic pressure tended to rise before the onset of ST segment depression. In contrast, during spontaneous episodes the reverse appeared to be the case in that the ST segment fell either at the same time or before the earliest change in pulmonary artery diastolic pressure. Pain was, however, a late feature that usually occurred after the maximum change in pulmonary artery diastolic pressure and ST segment had been reached. These results concur with previous findings in the catheterisation laboratory. ${ }^{4}$ After the end of exercise the pulmonary artery diastolic pressure tended to revert to normal much more rapidly than the ST segment. Alterations in myocardial perfusion and metabolism may take up to 20 minutes to return to normal after the end of an anginal episode. ${ }^{15}$ Left ventricular function has returned to normal before the myocardial cells have fully recovered their normal metabolic state.

Atrial pacing has been used to study myocardial ischaemia, particularly in patients with poor exercise tolerance. ${ }^{1617}$ The response of the left ventricle to atrial pacing has been variously reported. Some have shown a consistent rise in left ventricular end diastolic pressure ${ }^{35617}$ while others have not. ${ }^{1618}$ The physiological response to atrial pacing differs from that during exercise. Circulating catecholamines increase with treadmill exercise but not with atrial pacing. ${ }^{19}$ These results suggest that although atrial pacing may precipitate ST segment depression in patients with coronary artery disease, the haemodynamic response of the left ventricle resembles that in controls. Our results did not accord with those of an earlier study that suggested that in patients with coronary disease the magnitude of change in left ventricular end diastolic pressure was proportional to the maximum heart rate achieved during atrial pacing. ${ }^{20}$

The use of a transducer tipped catheter avoided the problems of a fluid filled system. Fluid filled systems suffer many disadvantages such as low resonant frequency and the need for a transducer perfusion unit with a constant zero reference point. ${ }^{21-23}$ Transducer tipped catheters have been used in the catheterisation laboratory for direct left ventricular pressure recordings, and a transducer tipped pulmonary artery pressure catheter has been used in association with an online computer, ${ }^{24}$ but this technique has not previously been applied to investigating the haemodynamics of myocardial ischaemia.

For the first time this technique has allowed the relation between ST segment changes and pulmonary artery diastolic pressure, which reflects changes in left ventricular end diastolic pressure, to be recorded in ambulant patients with coronary artery disease.

We thank the Clinical Research Committee of the National Heart and Chest Hospitals for financial support.

\section{References}

1 Müller O, Rørvik K. Haemodynamic consequences of coronary heart disease with observations during anginal pain and on the effect of nitroglycerine. Br Heart $\mathcal{F}$ 1958; 20: 302-10.

2 Chierchia S, Lazzari M, Freedman SB, Brunelli C, Maseri A. Impairment of myocardial perfusion and function during painless myocardial ischaemia. $\mathcal{f} \mathrm{Am}$ Coll Cardiol 1983; 1: 924-30.

3 Friesinger GC, Conti CHR, Pitt B. Observations on left ventricular pressure during angina pectoris [Abstract]. Circulation 1967; 36 (suppl 11): 115.

4 Parker JO, Di Giorgi S, West RO. Haemodynamic study of acute coronary insufficiency precipitated by exercise. Am f Cardiol 1966; 17: 470-83.

5 Rickards AF, Seabra-Gomes R. Observations on the effect of angina on the left ventricle with special reference to diastolic behaviour. Eur $\mathcal{f}$ Cardiol 1978; 7 (suppl): 213-38.

6 Linhart JW. Pacing induced changes in stroke volume in the evaluation of myocardial function. Circulation 1971; 43: 253-61.

7 Levy RD, Cunningham D, Shapiro LM, Wright C, Mockus L, Fox KM. Continuous ambulatory pulmonary artery pressure monitoring: a new method using a transducer tipped catheter and a simple recording system. Br Heart f 1986; 55: 336-43. 
8 Bruce RA, Blackman JR, Jones JW, Strait G. Exercise testing in adult normal subjects and cardiac patients. Pediatrics 1963; 32: 742-56.

9 Guazzi M, Polese A, Fiorentini C, Magrini F, Olivari MT, Bartorelli C. Left and right heart heart haemodynamics during spontaneous angina pectoris. Comparison between angina with ST segment depression and angina with ST segment elevation. Br Heart $\mathcal{F}$ 1975; 37: 401-13.

10 Figueras J, Singh B, Ganz W, Charuzi Y, Swan H. Mechanism of rest and nocturnal angina: observations during continuous haemodynamic and electrocardiographic monitoring. Circulation 1979; 59: 955-68.

11 Rackley CE, Russell RO Jr, Moraski RE, Mantle JA. Recent advances in haemodynamic studies in patients with acute myocardial infarction. In: Yu PN, Goodwin JF, eds. Progress in cardiology. Vol. 5 Philadelphia: Lea and Febiger, 1976: 201-26.

12 Scheinman M, Evans GT, Weiss A, Rapaport E. Relationship between pulmonary artery end diastolic pressure and left ventricular pressure in patients in shock. Circulation 1973; 47: 317-24.

13 Kaltman AJ, Herbert WH, Conroy RJ, Kossman CE. The gradient in pressure across the pulmonary vascular bed during diastole. Circulation 1966; 34: 377-84.

14 Berndt T, Fitzgerald J, Harrison D, Schroeder J. Haemodynamic changes at the onset of spontaneous versus pacing-induced angina. Am $\mathcal{F}$ Cardiol 1977; 39: 784-8.

15 Selwyn AP, Allan RM, Fox KM, Horlock P, O'Brian $\mathrm{H}$, Maseri A. Prolonged myocardial ischaemia in angina [Abstract]. Circulation 1981; 64 (suppl 4): 82.

16 Sowton GE, Balcon R, Cross D, Frick MH. Mea- surement of the angina threshold using atrial pacing. A new technique for the study of angina pectoris. Cardiovasc Res 1967; 1: 301-7.

17 Parker JO, Chiong MA, West RO, Case RB. Sequential alterations in myocardial lactate metabolism, ST segments and left ventricular function during angina induced by atrial pacing. Circulation 1969; 40: 113-31.

18 O'Brien K, Higgs L, Glancy D, Epstein S. Haemodynamic accompaniments of angina-a comparison during angina induced by exercise and by atrial pacing. Circulation 1969; 39: 735-43.

19 Dominiak P, Delius W, Grobecker H. Changes in plasma catecholamine concentrations in patients with coronary artery disease during pacing and physical exercise. Clin Cardiol 1985; 8: 77-81.

20 Balcon R, Wilkinson R, Walsh W, Rickards A. Criteria for the validity of the rise in left ventricular enddiastolic pressure following pacing induced angina. [Abstract]. Am $\mathcal{F}$ Cardiol 1974; 33: 124.

21 Ikram H, Richards AM, Hamilton EJ, Nicholls MG. Continuous recording of pulmonary artery pressure in unrestricted subjects. Br Heart $\mathcal{F} 1984$; 51: 421-6.

22 Grossman W. Pressure measurement. In: Grossman W, ed. Cardiac catheterisation and angiography. 2nd ed. Philadelphia: Lea and Febiger, 1980: 103-15.

23 Saumarez $\mathrm{RC}$ and Ikram $\mathrm{H}$ et al. Continuous recording of pulmonary artery pressure [Letters]. $\mathrm{Br}$ Heart $\mathfrak{f}$ 1985; 53: 581-4.

24 Nathan AW, Perry SG, Cochrane T, Banim SO, Spurrell RAJ, Camm AJ. Ambulatory monitoring of pulmonary artery pressure. A preliminary clinical evaluation. Br Heart $\mathcal{f}$ 1983; 49: 33-7. 